\title{
Awareness of Infection Control Protocols Among Clinical Dental Students and Interns in South Indian Universities
}

\author{
Aniruddh Menon ${ }^{1}$ and Dhanraj Ganapathy ${ }^{2}$ \\ ${ }^{1}$ ADepartment of Prosthodontics, Saveetha Dental College, Saveetha Institute \\ of Medical and Technical Sciences, Saveetha University, Chennai-77 \\ ${ }^{2}$ Professor and Head of Department, Department of Prosthodontics, Saveetha Dental College, \\ Saveetha Institute of Medical and Technical Sciences, Saveetha University, Chennai-77, India
}

\begin{abstract}
Infection control is the discipline concerned with preventing nosocomial or health care associated infections, a practical sub-discipline of epidemiology. It is essential, though often under-recognized and under-supported, part of the infrastructure of health care. The major aspects looked at in this study are infection control and water lines protocol. The study was performed with the help of a questionnaire prepared based on the latest CDC and OSHA guidelines. In this study an average of correct responses was 56\% whereas 51\% was the average of incorrect responses. There was a vast variation in the ideologies regarding the infection control protocols among the dental students. This was correlated to a more practical and lesser theoretical knowledge amongst the students. Dental students of south India have a good awareness of various infection control protocols and water lines maintenance protocols. There is still a lot of room for improvement by conducting more workshops, CDE programmes and seminars to further spread knowledge regarding the advancements in the above mentioned protocols. This will go a long way in improving the quality and standard of dental care in the country.
\end{abstract}

KEY WORDS: INFECTION CONTROL, WATERLINES, STUDENTS, SOUTH INDIA, NEEDLE STICK.

\section{INTRODUCTION}

Dentistry as a profession has grown over the past 150 years and the improvement in skills and variety of treatment procedures has grown by leaps and bounds. Present day advancements have seen an astronomical growth in efficiency as well as quality in dental work; for example, a patient can get a complete denture, a crown, a fixed partial denture and much more in just a day's

\section{ARTICLE INFORMATION}

*Corresponding Author:dhanraj@saveetha.com

Received 15th June 2020 Accepted after revision 10th August 2020 Print ISSN: 0974-6455 Online ISSN: 2321-4007 CODEN: BBRCBA

Thomson Reuters ISI Web of Science Clarivate Analytics USA and Crossref Indexed Journal

\section{Clarivate}

NAAS Journal Score 2020 (4.31) SJIF: 2020 (7.728)

A Society of Science and Nature Publication,

Bhopal India 2020. All rights reserved.

Online Contents Available at: http//www.bbrc.in/

Doi: $h$ ttp://dx.doi.org/10.21786/bbrc/13.7/52 time. There have also been many treatment practices and materials that have become obsolete over time but one aspect of dental practice that has always remained the same was the transmission of infection between the patient, operator and the dental assistant.

This where the role of organisations such as the CDC and OSHA are indispensable as these organizations have taken immense efforts to lay down safety measures that benefits both the practitioner as well as the patient. One of the strategic goals of the Division of Oral Health of the Centers for illness management and prevention (CDC), Atlanta, is to eliminate causes of illness transmission in dental health care settings. Their role is to provide evidence based information and proposals relating to dental infection management by maintenance of these high standards of infection management guidelines

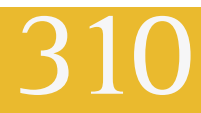


in practice.(of Health and Committee, 2016)The CDC printed infection management recommendations for medical specialty initial in 1986 (Centers for Disease Control, 1986)and subsequently in 1993.(Kohn et al., 2004) These pointers were developed partially in response to reports relating to 9 clusters of viral hepatitis virus (HBV) transmission to patients from infected dental health care suppliers (DHCPs) throughout the 70's and 80's.(Kohn et al., 2003) During those periods, there were high prevalence of markers of past HBV infection among medical personnel and other associates in Nursing and oral surgeons(Cleveland, 1996) and reports of transmission of HIV from an infected dentist to patients. (Ciesielski et al., 1992)

Serologic proofs of past HBV infection among U.S. dentists decreased from pre-vaccine levels of $14 \%$ in 1972 to $9 \%$ in 1992. In the year 2007, there was a report of transmission of HBV from one infected patient to another in a dental surgeon's workplace. (Redd et al., 2007) In 2009, 5 cases of acute viral hepatitis were diagnosed among 3 patients and 2 volunteers at a dental clinic in West Virginia. (Holmberg, Suryaprasad and Ward, 2012) During an investigation, the CDC investigators and West Virginia public health officials identified multiple infection control breaches that had taken place, but improper documentation did not allow for linkage of specific breaches with transmission. There are no documented cases of patient-to-patient transmission of HIV or hepatitis $\mathrm{C}$ virus during a dental setting. There are massive declines in HBV infection and rare occurrences of blood-borne infectious agent transmissions in dental settings owing touse of the HBV vaccines and enhanced use of universal precautions. (Quinley et al., 1989)

Identifying all transmission routes of infectious agents may be a key part of any infection management protocol. In dentistry special care is to be given to those instruments that are not routinely placed into the patient's mouth and are otherwise not routinely disinfected due to their design or another thought. (Biofilms, 2000; Kohn et al., 2004). Dental instruments are classified broadly into three categories depending on the risk of transmitting infection. Critical instruments are grouped as those used to penetrate soft tissue or bone, or come directly into the bloodstream. They should always be sterilized after each time they are used. Sterilization is achieved by autoclaving, dry heat, chemical vapour. Critical instruments include forceps, scalpels, bone chisels, scalers (both ultrasonic as well as hand scalars) and surgical burs. Semi-critical instruments are those that do not penetrate soft tissues or bone but contact mucous membranes or non-intact skin, such as mouth mirrors, impression trays and amalgam condensers.

These devices also should be sterilized after each use just like the critical instruments. Where sterilization is not a feasible option, high-level disinfection is appropriate. Non-critical instruments are those that do not come into contact with any other part of the body other than intact skin, for example x-ray heads, BP cuffs and stethoscopes. Such devices have relatively very low chances of transmitting infection; and hence, may be reprocessed between patients by intermediate or lowlevel disinfection. An intermediate-level disinfectant is EPA-registered as a "hospital disinfectant" with a label for "tuberculocidal" activity, like phenolics, chlorine-containing compounds). (Biofilms, 2000) The tuberculocidal activity is given to be used as a benchmark to measure germicidal potency.

Figure 1: Bar Graph shows distribution of responses received for each question included in the present study.

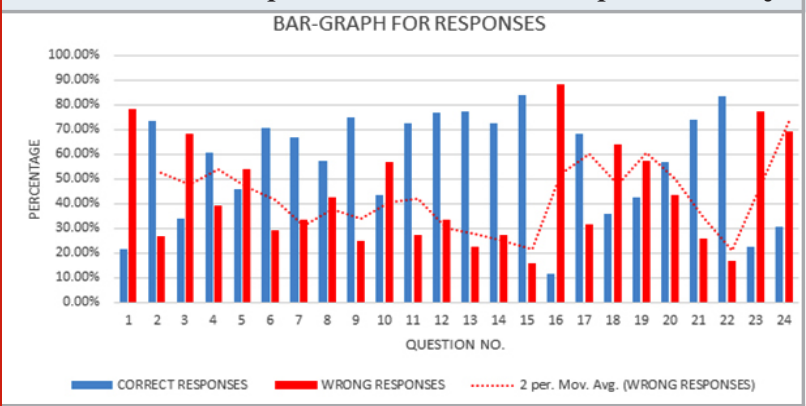

Figure 2: Pie Chart Shows Distribution of answers received for question on factor dependent on deciding precautions before procedures. The most important factor that has been voted for is risk of transmission (37\%) and the least important factor is quality of precautions (7\%)

3. Most Important Factor in Deciding if one of our patients needs precautions before procedures
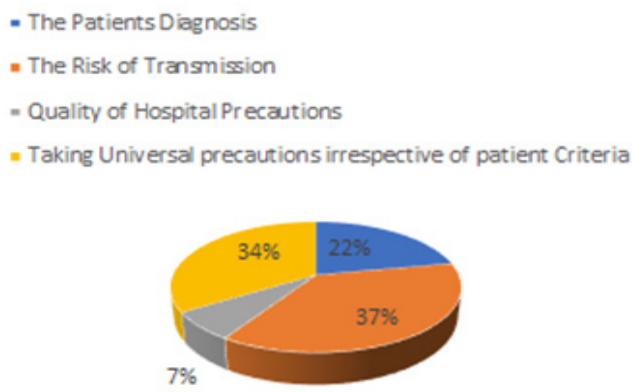

Figure 3: Pie Chart Shows Distribution of answers received for question on hand hygiene prior to wearing gloves. 70\% of the study population voted that hand hygiene needs to be practiced every time before wearing gloves and 4\% voted it was not mandatory.

6. Hand Hygiene Practice Prior to Wearing Gloves are

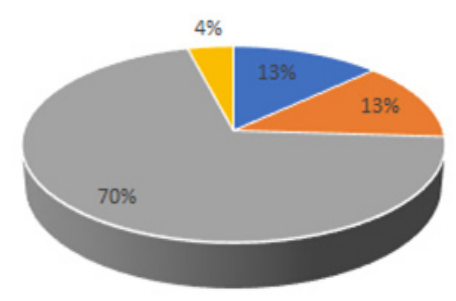

- Needed only when hands are visibly solid or dirty

- Needed only when working on patients with obvious infection

- Needed everytime 
Dental hand items (specifically high-speed drills), air/ water syringes and ultrasonic scaler units connected to dental units by a network of small-bore plastic tubes through that water and air are propelled to activate or quiet down the instruments. Hydrodynamics shows that the water column within a small lumen moves within the centre of the tube, leaving a thin layer of liquid just about undisturbed on the walls. Repeated semi-permanent water stagnations during the nights, weekends and holidays combined with high temperatures creates favourable conditions for water flora to form tenaciously adherent microbial colonies. Some dental unit waterlines that are in use for several years could be coated with a biofilm that are visible to the eye, clogs the small-bore tube and gives the water a foul odour. (Miller (Microbiologist) and Palenik, 2018) Biofilm is outlined as a mass and they simply form at anyplace there is a damp non sterile atmosphere.(Yüzbasioglu et al., 2009) Bacteria and yeasts from the biofilms may produce aerosols in the dental surgery. Bacterial species such as Pseudomonas aeruginosa, Pseudomonas cepacia, Legionella pneumophila and mycobacterium chelonae have been identified in biofilms.

Figure 4: Pie Chart Shows Distribution of answers received for question on cleaning waterlines of dental unit. Out of the study population $72 \%$ cleaned the waterlines of their dental unit while $28 \%$ did not.

14. Do you clean the waterlines of your dental unit - Yes - No

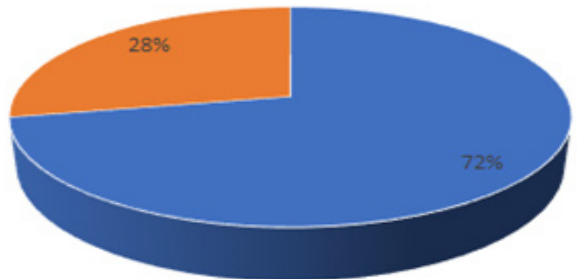

Figure 5: Pie Chart Shows Distribution of answers received for question on flushing the water lines at the start of the day. $84 \%$ of participants flushed the dental air/water lines at the start of each day whereas $16 \%$ did not follow the same.

15. Do you flush the dental air/water lines at the start of each day

- Yes - No

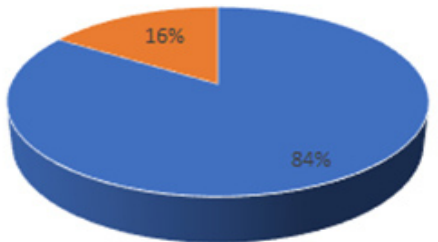

Numerous practice guidelines have been put in place by the CDC and OSHA to help benefit the practitioners and the patients alike. While most of these are implemented at a Dental School level training, it is not integrated as a part of the curriculum. Hence the infection control practices are followed more mechanically than with meticulous thought and implementation. This survey is conducted to assess the knowledge and awareness not only of the protocols followed, but also of the science and rationale behind their usage. This study was aimed to check the awareness of the dental students of Chennai and other South Indian Universities regarding the latest guidelines in infection control protocols.

Previously our department has published extensive research on various aspects of prosthetic dentistry ('Evaluation of Corrosive Behavior of Four Nickelchromium Alloys in Artificial Saliva by Cyclic Polarization Test:An in vitro Study', 2017; Ganapathy, Kannan and Venugopalan, 2017; Jain, 2017a, 2017b; Ranganathan, Ganapathy and Jain, 2017; Ariga et al., 2018; Gupta, Ariga and Deogade, 2018; Anbu et al., 2019; Ashok and Ganapathy, 2019; Duraisamy et al., 2019; Varghese, Ramesh and Veeraiyan, 2019), this vast research experience has inspired us to research about awareness of infection control protocols among clinical dental students and interns in south indian universities.

\section{MATERIAL AND METHODS}

This study was performed with the help of a questionnaire consisting of 24 questions and was prepared based on the guidelines of the CDC and OSHA as shown below in FIG 1A,B,C. The questionnaire was given to the 3rd BDS and CRI's students of multiple Dental Colleges in South India. The questionnaire was prepared with the help of google forms and the statistical analysis was done.

\section{Questionnaire}

1 The standard Universal Precaution: should be used on:

2. The 'Chain of Infection' involves:

3 According to vouch most important factor in deciding if one of our patients need:

precautions beyond Standard procedures are:

4 You can protect yourself from transmission of Hepatitis B. Hepatitis C and HIV by:

5. Hand Hygiene is a vital part of Infection Control because:

6 Hand byriene practice prior to wearing gloves are:

7. Ways to prevent the spread of the skin infection MRSA is the general community include?

8. Good "cough etiquette" means?

9 Is it ok to come to work when you're sick and infectious, because the hospice mission is more important than anything?

10. What are the first things you need to know about Infection Control?

11. What is the water temperature you should wash your hands with? 
12 What is the first basic element of Infection Control?

13. Which one of the following is PPE?

14 Do you clean the water lines of your dental unit?

15 Do you flush the dental water lines at the start of each day?

16 If so, for how much duration?

17 Do vou flu:h che dental air water lines in between patients appointment:?

18 If yes, for how much duration?

19 How many steps are involved in the spray-wipespray technique?

20 Do hand lotions: affect the integrity of gloves?

21 Does wearing gloves replace the need for hand washing ?

22 Are you aware of management of blood contaminated instruments?

23 A needle stick injury occurring with s patient with acute HBV infection

necessitate:?

24. A needlestick injury occurring with a patient who has AIDS necessitates?

\section{RESULTS AND DISCUSSION}

A total of 120 participants enrolled in the study. The responses of all 24 questions are depicted in Fig 2 . The vast variation in ideas among practitioners regarding infection control questions can be seen from the variations in the responses Some of the results of individual questions that had shown a wide variation in assumptions among the practitioners are shown in the figures below

Figure 6: Pie Chart Shows Distribution of answers received for question on how long they flush the water lines at the start of the day. $9 \%$ of the study population were aware that 2 minutes was the ideal duration to flush their water lines at the start of the day whereas the remaining $91 \%$ did not know the correct duration.

16. How long do you flush the air/water lines

- 15 seconds $=30$ seconds $=1$ minute $=2$ minutes $=$ Do not flush

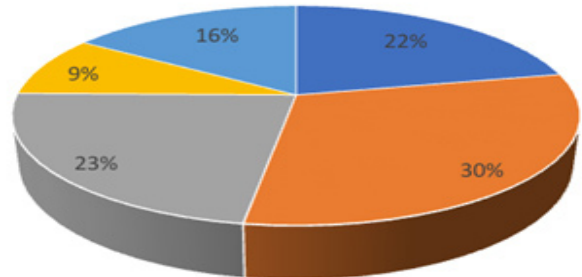

Dental practitioners are especially at high risk of infection by aerosol, blood pathogens, saliva, as they are continually work closest to the patients face and oral cavity and hence exposed to blood and saliva mixed with blood, and may even suffer needle punctures.(Association and Center, 2001) Dental institutes are responsible for providing appropriate training of dental students toward infection control measures.(Mp, 2016) A wide variety of bacteria, viruses and other pathogens can cause a wide variety of diseases such as Hepatitis B,C,D , HIV, tuberculosis etc. within dental clinical environment if the dental student didn't have enough knowledge, training and education about infection control. (Singh et al., 2011) The spread of infection can be limited by proper awareness of infection control protocols and use of appropriate personal protective equipment and timely vaccination.(Kushiyama, Shimazaki and Yamashita, 2009; Rahman et al., 2013).

Figure 7: Pie Chart Shows Distribution of answers received for question on flushing air/waterlines in between appointments. $68 \%$ of the study population flushed the air and water lines between appointments in contrast to remaining 32\% that did not.

17. Do you flush the air or water lines in between appointments

- Yes - No

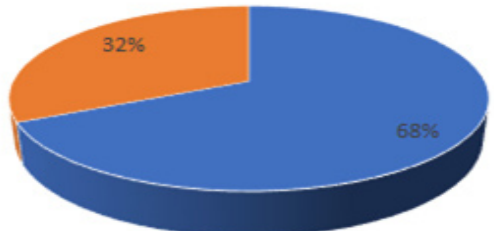

Figure 8: Pie Chart Shows Distribution of answers received for question on duration of flushing air/waterlines. 30\% of the study population were aware of the appropriate time for flushing air/waterlines whereas $70 \%$ were not.

18. How long do you flush your air/water lines in between appointments

- 15 seconds $=30$ seconds $=1$ minute $=2$ minutes $=$ Do not flush

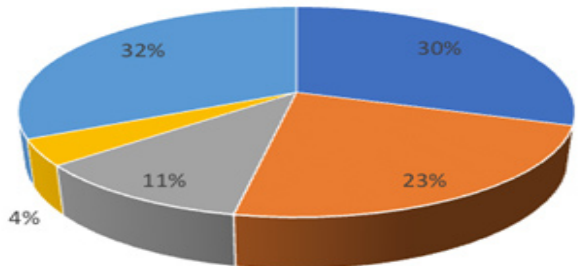

Also the post exposure management of the disease is also very important in minimizing the risk to the practitioner. (Ojulong, Mitonga and Iipinge, 2013) The study can be divided into awareness on infection control, waterlines maintenance and infectious diseases and blood products. A majority of the students (73\%) were aware about the various modes of spread and basic requirements to prevent spread infectious diseases. While $70 \%$ of the students knew the proper hand hygiene protocol, only a contrasting $42.8 \%$ of the students knew the actual reason behind the need for proper hand washing. Only $43 \%$ of the students knew that hand-washing was the primary factor in infection control, instead a greater majority 
assumed it was biohazards and disinfection. This is due to the fact of the common misconception that the cause of infection isn't the doctor or the patient, but instead the objects. 77\% of the participants answered correctly as to what comprises personal protective equipment, this can be attributed to the good amount of clinical exposure they are given at an undergraduate level.

Figure 9: Pie Chart Shows Distribution of answers received for question on need for wearing gloves. 74\% of the students believed that wearing gloves replaced the need for hand washing and $26 \%$ did not feel the same.

21. Does wearing gloves replace the need for hand washing

- Yes = No

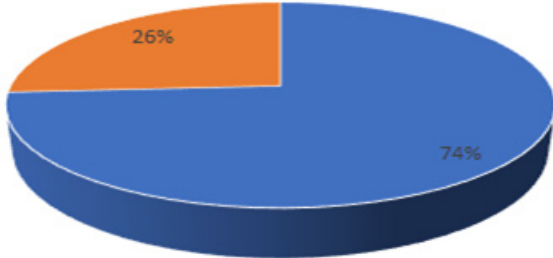

Figure 10: Pie Chart Shows Distribution of answers received for question on necessities for needle stick injury from a patient with HBV. Out of the study population, 51\% said they needed hepatitis B vaccine whereas only $23 \%$ said HBIG is required.

23. A needle stick injury occurring with a patient with active HBV Infection necessities

- Hepatitis B Vaccination - HAART regimen - HBIG administration - NONE

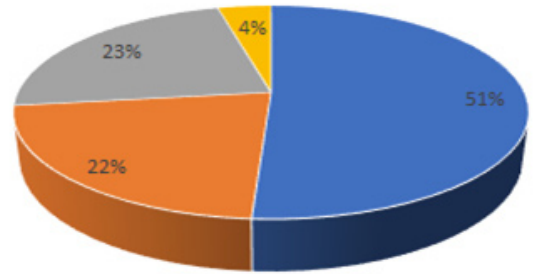

The odds of a patient experiencing an infection could also be related to the quality of water present in the water lines. If the colony forming units(CFU) exceeds the recommended number as per the ADA guidelines of $500 \mathrm{CFU} / \mathrm{ml}$, there is a high chance of risk of infection(Holmberg, Suryaprasad and Ward, 2012).It is important for the dental students to be aware of all the above said protocols. As our education system does not focus on the awareness of these protocols, through the four years of their undergraduate education they tend to forget about this aspect of dental practice. This is the reason for a lot of mishaps and problems occurring in the private dental practices.

The majority of the participants flushed and cleaned their water lines both at the beginning of the day as well as between patients. However, only a meagre 9\% and 30\% of the participants knew the correct protocol wherein the water lines had to be flushed for 2 minutes and 15 seconds at the start of the day and between patient appointments respectively. This maintains the CFU $<500$ $\mathrm{CFU} / \mathrm{ml}$ to minimize the risk of infections to the patient. (Singh et al., 2011) With reference to the management of sharps and needlestick injuries, the participants showed good awareness regarding the requirement of a post-exposure prophylaxis protocol for needle stick injuries, but a majority (77\% and 69\%) didn't know the correct drug to be administered in a scenario of a needle stick injury from a patient suffering from Hepatitis B or AIDS,.

In this study an average of correct responses was 56\% whereas 51\% was the average of incorrect responses. In paralleling studies conducted in Saudi Arabia, the dental students showed a lower percentage of awareness of only 51\%,(Rahman et al., 2013) as well as in Qatar the dental students showed a lower percentage of awareness of 44.84\%.(Ibrahim and Elshafie, 2016) However, the students of Namibia showed a particularly high awareness of 73\%.(Ojulong, Mitonga and Iipinge, 2013). The wide range of fluctuations in the awareness of basic infection control protocols as observed in this survey seem to be present universally across many countries as well. This could be attributed to the fact that in the majority of the dental schools, the infection control protocols are just blindly followed as a part of the clinic practice and there is no academic integration for improvement of the student knowledge regarding the science and logic behind it.

This could be improved by taking meticulous efforts in separately training the dental practitioners and educating them about the serious implications of the same. It is important to reinforce the fact that however long the practitioner has been successful over time, it just takes one little negligence to affect the health of either the practitioner and/or the patient. The role of various local dental bodies is also supposed to focus towards enhancing the knowledge of the practitioners with respect to the infection control practices, apart from the regular programs that only aim in improving skills or demonstrating advanced procedures alone.

Figure 11: Pie Chart Shows Distribution of answers received for question on necessities for needle stick injury from a patient with HIV. Out of the study population, $31 \%$ said that HAART treatment is required and 14\% said HBIG is required.

24. A needle stick injury occurring with a patient who has AIDS necessities

- HBIG Administration - HIVVaccination - HAART = Antiviral drugs

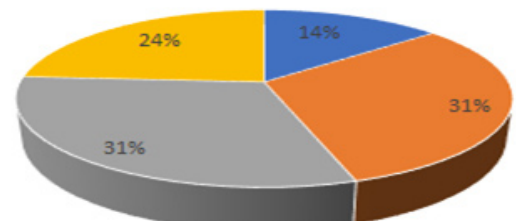




\section{CONCLUSION}

Dental students of south India have a fairly good awareness of various infection control protocols and water lines maintenance protocols. However, a wide variation among the ideas and a lot of misconceptions regarding the infection control protocols show that there is still a lot of room for improvement by conducting more number of workshops, continuous dental education programmes and seminars to further spread knowledge regarding the advancements in the above mentioned protocols. This will go a long way in improving the quality and standard of dental care in the country.

\section{ACKNOWLEDGEMENTS}

No Acknowledgements

\section{Conflict of Interest: No Conflict of Interest}

\section{REFERENCES}

Anbu, R. T. et al. (2019) 'Comparison of the Efficacy of Three Different Bone Regeneration Materials: An Animal Study', European journal of dentistry, 13(1), pp. 22-28.

Ariga, P. et al. (2018) 'Determination of Correlation of Width of Maxillary Anterior Teeth using Extraoral and Intraoral Factors in Indian Population: A Systematic Review', World Journal of Dentistry, 9(1), pp. 68-75.

Ashok, V. and Ganapathy, D. (2019) 'A geometrical method to classify face forms', Journal of oral biology and craniofacial research, 9(3), pp. 232-235.

Association, A. D. and Center, A. D. A. H. P. R. (2001) Future of dentistry: today's vision, tomorrow's reality. American Dental Association.

Biofilms, W. (2000) 'Waterborne biofilms and dentistry: the changing face of infection control', Journal . cdaadc.ca, 66, pp. 539-541.

Centers for Disease Control (1986) 'Recommended infection-control practices for dentistry', MMWR. ci.nii. ac.jp, 35, pp. 237-241.

Ciesielski, C. et al. (1992) 'Transmission of human immunodeficiency virus in a dental practice', Annals of internal medicine. American College of Physicians, 116(10), pp. 798-805.

Cleveland, J. L. (1996) 'HEPATITIS B VACCINATION AND INFECTION AMONG U.S. DENTISTS, 1983-1992', The Journal of the American Dental Association. Elsevier, 127(9), pp. 1385-1390.

Duraisamy, R. et al. (2019) 'Compatibility of Nonoriginal Abutments With Implants: Evaluation of Microgap at the Implant-Abutment Interface, With Original and Nonoriginal Abutments', Implant dentistry, 28(3), pp. 289-295.

Evaluation of Corrosive Behavior of Four Nickel- chromium Alloys in Artificial Saliva by Cyclic Polarization Test:An in vitro Study' (2017) World Journal of Dentistry, 8(6), pp. 477-482.

Ganapathy, D. M., Kannan, A. and Venugopalan, S. (2017) 'Effect of Coated Surfaces influencing Screw Loosening in Implants: A Systematic Review and Meta-analysis', World Journal of Dentistry, 8(6), pp. 496-502.

Gupta, P., Ariga, P. and Deogade, S. C. (2018) 'Effect of Monopoly-coating Agent on the Surface Roughness of a Tissue Conditioner Subjected to Cleansing and Disinfection: A Contact Profilometric Study', Contemporary clinical dentistry, 9(Suppl 1), pp. S122S126.

of Health, U. S. D. and Committee, H. S. O. H. C. (2016) 'US Department of Health and Human Services oral health strategic framework, 2014--2017', Public health reports. SAGE Publications Sage CA: Los Angeles, CA, 131(2), pp. 242-257.

Holmberg, S. D., Suryaprasad, A. and Ward, J. W. (2012) 'Updated CDC Recommendations for the Management of Hepatitis B Virus-Infected Health-Care Providers and Students', MMWR. Recommendations and reports: Morbidity and mortality weekly report. Recommendations and reports / Centers for Disease Control. Centers for Disease Control \&t Prevention (CDC), 61(3), pp. 1-12.

Ibrahim, A. A. and Elshafie, S. S. (2016) 'Knowledge, awareness, and attitude regarding infection prevention and control among medical students: a call for educational intervention', Advances in medical education and practice. Dove Press, 7, p. 505.

Jain, A. R. (2017a) 'Clinical and Functional Outcomes of Implant Prostheses in Fibula Free Flaps', World Journal of Dentistry, 8(3), pp. 171-176.

Jain, A. R. (2017b) 'Prevalence of Partial Edentulousness and Treatment needs in Rural Population of South India', World Journal of Dentistry, 8(3), pp. 213-217.

Kohn, W. G. et al. (2003) 'Guidelines for infection control in dental health-care settings-2003'. stacks.cdc.gov. Available at: https://stacks.cdc.gov/view/cdc/7093.

Kohn, W. G. et al. (2004) 'COVER STORY Guidelines for infection control in dental health care settings-2003', The Journal of the American Dental Association. Elsevier, 135(1), pp. 33-47.

Kushiyama, M., Shimazaki, Y. and Yamashita, Y. (2009) 'Relationship Between Metabolic Syndrome and Periodontal Disease in Japanese Adults', Journal of periodontology. Wiley Online Library, 80(10), pp. 1610-1615.

Miller (Microbiologist), C. H. and Palenik, C. J. (2018) Infection Control and Management of Hazardous Materials for the Dental Team. Elsevier Health 
Sciences.

Mp, S. K. (2016) 'Knowledge, attitude, and practices regarding infection control among undergraduate dental students', Asian J Pharm Clin Res, 9(1), pp. 220-224. Ojulong, J., Mitonga, K. H. and lipinge, S. N. (2013) 'Knowledge and attitudes of infection prevention and control among health sciences students at University of Namibia', African health sciences. ajol.info, 13(4), pp. 1071-1078.

Quinley, E. D. et al. (1989) 'The air-water syringe: contamination and disinfection', Quintessence international . europepmc.org, 20(12), pp. 911-916.

Rahman, B. et al. (2013) 'Attitudes and practices of infection control among senior dental students at college of dentistry, university of Sharjah in the United Arab Emirates', European journal of dentistry. Thieme Medical and Scientific Publishers Private Ltd., 7(S 01), pp. S015-S019.

Ranganathan, H., Ganapathy, D. M. and Jain, A. R. (2017) 'Cervical and Incisal Marginal Discrepancy in Ceramic Laminate Veneering Materials: A SEM Analysis', Contemporary clinical dentistry, 8(2), pp. 272-278.

Redd, J. T. et al. (2007) 'Patient-to-Patient Transmission of Hepatitis B Virus Associated with Oral Surgery', The Journal of infectious diseases. Oxford Academic, 195(9), pp. 1311-1314.

Singh, A. et al. (2011) 'Knowledge, Attitudes, and Practice Regarding Infection Control Measures Among Dental Students in Central India', Journal of dental education. Wiley Online Library, 75(3), pp. 421-427. Varghese, S. S., Ramesh, A. and Veeraiyan, D. N. (2019) 'Blended Module-Based Teaching in Biostatistics and Research Methodology: A Retrospective Study with Postgraduate Dental Students', Journal of dental education, 83(4), pp. 445-450.

Yüzbasioglu, E. et al. (2009) 'A survey of cross-infection control procedures: knowledge and attitudes of Turkish dentists', Journal of applied oral science: revista FOB. USP, 17(6), pp. 565-569. 